\title{
Development of Indoor Aquaponics Control System Using a Computational Thinking-based Convergence Instructional Model
}

\author{
Sook-Young Choi ${ }^{1}$, A-Mi Kim ${ }^{2, *}$ \\ ${ }^{1}$ Department of Information Security, Woosuk University, South Korea \\ ${ }^{2}$ Department of Liberal Art, Woosuk University, South Korea
}

Copyright $\odot 2019$ by authors, all rights reserved. Authors agree that this article remains permanently open access under the terms of the Creative Commons Attribution License 4.0 International License

\begin{abstract}
Attempts have been made to introduce computational thinking (CT) into STEAM education as part of the training of creative and convergent talents. In this study, we designed a CT-based convergence instructional model for creative and integrated education and we developed a system to solve real-world problems using this model. The system developed as a product for convergence education is an indoor Aquaponics model. The Aquaponics model is a circulating model that takes advantage of fish culture and hydroponics cultivation and has recently been highlighted for its eco-friendliness, productivity improvement, and labor reduction. However, it has the disadvantage that continuous monitoring of growth and cultivation environment is necessary. Therefore, with the instructional model we designed, we implemented a remote automatic control using the IoT (Internet of things) to solve the problem of manual monitoring of the Aquaponics model. The results of this study are expected to be the basis of convergence education, leading to creative real-world problem solving through the fusion of science, engineering, and technology, based on CT.
\end{abstract}

Keywords Computational Thinking, Convergence, IoT, Aquaponics, Instructional Model

\section{Introduction}

Today, the key to convergence within and between industries is software (SW). SW combines ICT technology as an infrastructure for all industries to create business models for new products and services. In particular, as the era of the Fourth Industrial Revolution is expected to come, there will be many changes in our society. In the industrial sector, high-tech industries based on ICT are expected to generate great benefits [1][2][3].

As the industrial landscape changes and the jobs demanded are different from those of the past, the competencies required for future talents are also changing. In the era of the Fourth Industrial Revolution of super-intelligence, super-convergence and complex problem solving capabilities are important. To this end, education to enhance the capacity of fusion thinking and creativity based on computational thinking (CT) should be provided [4][5]. CT education can connect comprehension of computing to other subjects or other social problems to create new things or solve problems [6].

In the field of science education, STEAM (Science, Technology, Engineering, Art, and Mathematics) has been introduced to meet these rapidly changing social changes and needs of learners. However, educational experts noted that STEAM education has not yet successfully integrated science, technology, engineering, and mathematics, but has provided them in a fragmentary way [7].

To overcome this issue, there have been attempts to introduce CT into STEAM. CT is becoming the key to the STEAM field, especially because computer science is a necessary part of the educational goals pursued in STEAM education.

In Korea and abroad, examples of convergence education applying CT are presented in subjects such as mathematics and science. In the study of Ham et al. [8],[20], CT-STEAM was developed, which is a new type of convergence talent education. The model uses a variety of computational devices to solve real-world complex problems from a converged perspective. Park \& Hwang [9],[21] studied the CT evaluation tool for STEAM education in science. However, compared to foreign countries, studies that apply CT to STEAM education are not yet being actively conducted in Korea.

This study considers problem solving based on CT for creative convergence education. To do this, we have 
designed a new CT-based convergence instructional model by analyzing existing related studies. In addition, we selected an Aquaponics model and analyzed its existing issue in solving real-world problems using the model. Based on the analysis results, we built a new Aquaponics model and developed a remote automated control system based on the Internet of things (IoT).

This study was conducted for students in the course of the gifted education programs attached to the university. Convergence education based on CT has a positive effect on students' interest and satisfaction in learning. Based on the results of this study, it is expected that various examples of CT-based convergence education will be derived.

\section{Related Works}

\subsection{CT and Convergence Education}

In 2006, J. M. Wing stated that not only computer scientists but all learners should be equipped with computing thinking ability in addition to basic learning ability of reading, writing, and counting [10]. Since her assertion, CT has been very popular, and nowadays almost everyone is expected to have basic computing skills in parallel with technology regardless of age. CT is being developed not only in computer science but in many other fields, and it is regarded as a basic learning ability to grow into a key talent in the digital society of the 21 st century. Hence, being a digital citizen requires students to possess CT skills as defined by ISTE and indicated in the "Framework for K-12 Science education" [11], [23].

Wing [12] defined CT as "a thought process that can analyze problems and format them in a form that can be effectively implemented by computers". Abstraction and automation are introduced as sub-components of CT. After that, many institutions defined the concept of CT and the operational definition of sub-elements in detail. Table 1 shows a comparison of CT definitions and components.

The convergent approach to education can not only achieve academic inquiry, but also deal with real-world problems in a multidimensional and in-depth manner, enabling more practical education [13],[24]. In Korea, STEAM education, called "convergence talent education", emphasizes the need for convergence education, and strives to implement it. The Korea Science and Creativity Foundation defined STEAM literacy as "the ability to solve complex problems using diverse knowledge" [14], [25][29][30][31].

The most important aspect in convergence education is to learn convergence thinking. CT is a basic skill that requires convergence thinking abilities and is a core concept of computer science education [12]. For a while, convergence education of science, technology, engineering, and mathematics (STEM) has been a hot topic of science education. Now, CT meets with STEM, which naturally becomes a form of IT convergence education.

Table 1. Computational Thinking Components

\begin{tabular}{|c|c|c|c|c|}
\hline Researchers & ISTE \& CSTA [15] & BBC [16] & Brennan \& Resnick [17] & Selby \& Woolard [18] \\
\hline $\begin{array}{c}\text { Computational } \\
\text { thinking } \\
\text { Components }\end{array}$ & $\begin{array}{ll}\text { - } & \text { Data collection } \\
\text { - } & \text { Data Analysis } \\
\text { - } & \text { Data Representation } \\
\text { - } & \text { Problem } \\
& \text { decomposition } \\
\text { - } & \text { Abstraction } \\
\text { - } & \text { Algorithm } \\
& \text { procedure } \\
\text { - } & \text { Automation } \\
\text { - } & \text { Simulation } \\
\text { - } & \text { Parallelism }\end{array}$ & $\begin{array}{ll}\text { - } & \text { Decomposition } \\
\text { - } & \text { Pattern } \\
& \text { recognition } \\
\text { - } & \text { Abstraction } \\
\text { - } & \text { Algorithms } \\
\text { - } & \text { Evaluating } \\
& \text { solutions }\end{array}$ & $\begin{array}{l}\text { - Computational } \\
\text { Concepts } \\
\text { - } \text { variable, loop } \\
\text { Computational } \\
\text { Practices } \\
\text { Being incremental } \\
\& \text { iterative, testing } \\
\& \quad \text { debugging, } \\
\text { reusing, abstracting } \\
\text { Computational } \\
\text { perspective } \\
\text { Expressing \& } \\
\text { questioning about } \\
\text { the technological } \\
\text { world }\end{array}$ & $\begin{array}{ll}\text { - } & \text { Abstraction } \\
\text { - } & \text { Decomposition } \\
\text { - } & \text { Algorithmic } \\
& \text { design } \\
\text { - } & \text { Evaluation } \\
\text { - } & \text { Generalization }\end{array}$ \\
\hline
\end{tabular}




\subsection{CT Model for Convergence Education}

Lee [19] suggested that CT-based STEAM education should be conducted, because it can provide a framework of convergence between each subject.

In a study conducted to apply $\mathrm{CT}$ in the field of science education, Park [20],[22] developed CT_STEAM_AT, which links specific action guidelines and learning objectives for CT, based on six practices in the US computer science curriculum (The College Board, 2013). In addition, Park has devised detailed elements and operational definitions of $\mathrm{CT}$ in order to apply $\mathrm{CT}$ in science education.

Ham et al.[8] developed CT-STEAM education programs for enhancing integrated thinking skills of elementary students, which is an instructional model that applied CT to STEAM education. According to the results of the study, the instructional model was effective in the preference of STEAM subjects, self-directed learning, and so on, making it necessary to apply it in subsequent researches and education.

Psycharis [6] presented a computational science education model that considers the viewpoints of CT and engineering design. In particular, their models are based on inquiry-based learning to improve existing STEM education and approach from the viewpoint of computational experiments and epistemology of engineering education. Meanwhile, Han \& Ryu [21] proposed a CT-centered model for creative computing, which is based on Google's CT learning. Fig. 1 shows these models.

When analyzing the problems in the models presented in Fig. 1, the one presented by Psycharis [6],[26] needs accurate understanding and analysis of the problem. The model did not include data collection and data analysis, even though they are important factors. The CT-STEAM education approach suggested by Ham et al. [8] is an instructional model for problem solving and simplifying complexity are identified considering CT to enhance convergence thinking, but it does not consider the engineering aspects pursued in STEAM education. The DDAA model presented by Han \& Ryu [21], [27] is a CT element-centered model, but it was not considered in terms of convergence education.

As described above, there have been a few of studies on CT models for convergence education so far, but each model has drawbacks. In this study, we developed a CT model for the convergence education by analyzing such problems of the existing systems and complementing them. In addition, there have been no cases of applying CT models to develop the indoor aquaponics system except this study [28].

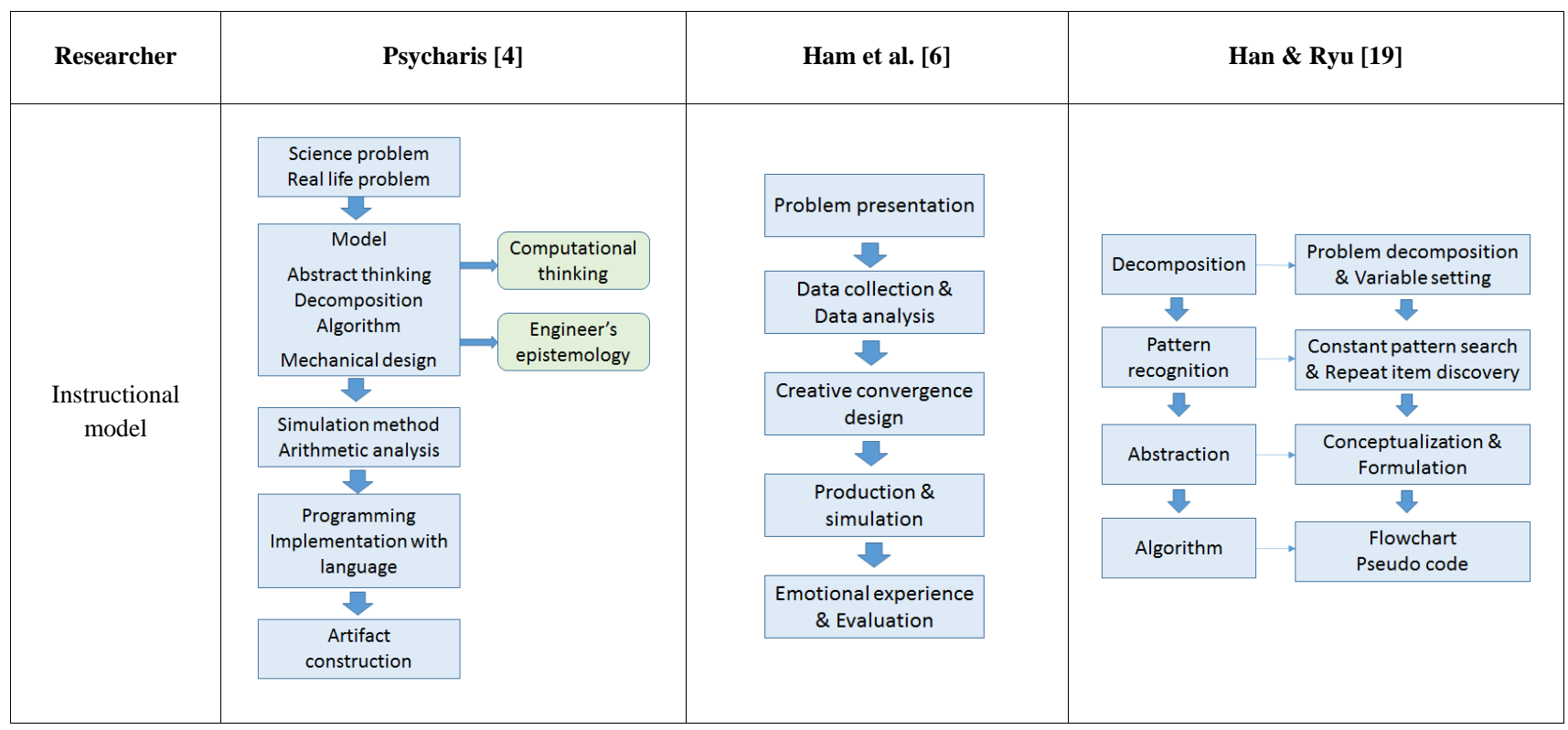

Figure 1. Instructional models for convergence education 


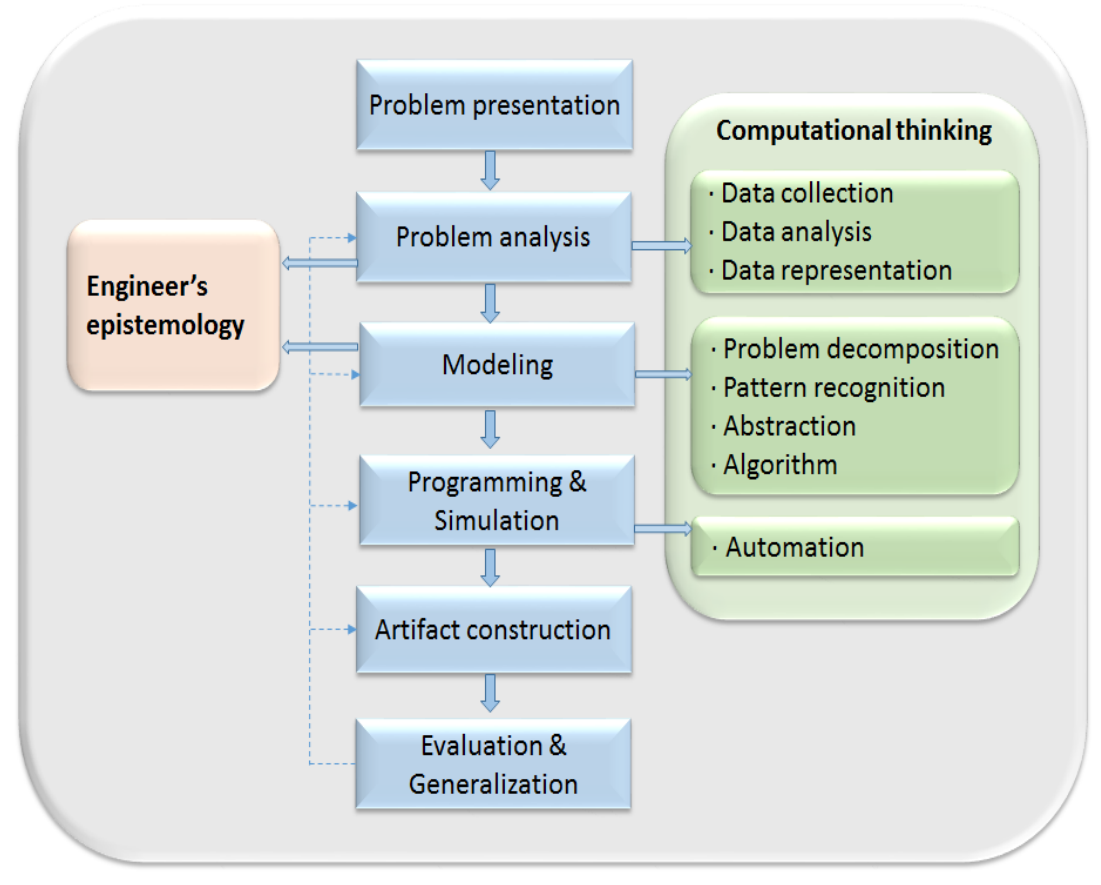

Figure 2. CT-based convergence instructional model

\section{Instructional Model for CT-based Convergence Education}

In this study, we designed an instructional model for convergence education by referring to the $\mathrm{CT}$ based teaching models presented in the related studies. We developed the model shown in Fig. 2, complementing the disadvantages of each model in the literature. The model proposed considers CT and engineering aspects.

First, if a problem is given, understanding and analyzing the problem is required. For this, data collection and data analysis are required. Moreover, the analyzed data are organized using graphics, tables, and pictures so that they can be easily understood. The relevant CT elements include data collection, data analysis, and data representation. The third step is the process of model design to solve the problem with the analyzed contents. In this modeling phase, the problem is decomposed into smaller problems that can be more easily solved, and the patterns repeatedly expressed to solve the problem are found. Here, the key elements that are essential for problem solving and simplifying complexity are identified. Based on these steps, an algorithm for problem solving is written. In the second and third step, students are encouraged to consider engineering aspects in the process of problem analysis and modeling. The fourth step involves implementing the algorithm using a programming language or tools. At this stage, debugging processes are required, as well as simulation processes to check whether the implemented model is performing well. Through this process, the output is generated. Students will then have time to present and share the generated outputs, and to communicate the difficulties and gains of knowledge and insights in the development process. In addition, they will take time to discuss what parts can be improved, or to expand on the basis of the output to produce other outcomes.

\section{Case Study}

This chapter describes the development of a real system using the CT-based convergence instructional model described in Chapter III.

\subsection{Problem Presentation}

Aquaponics models were selected as topics for convergence education. Aquaponics is a circulating model that takes advantage of fish culture and hydroponics cultivation and is characterized by eco-friendliness, productivity improvement, and labor saving. However, there is the problem that manual monitoring must be continuously performed to confirm that the cultivation system operates normally, such as sufficient water supply and circulation, and proper temperature maintenance. Accordingly, this study aims at building an indoor ornamental model and developing a control system to reduce the inconvenience of manual monitoring.

\subsection{Problem Analysis}

This step is a problem analysis step. Fig. 3 shows the step. It involves expressing the characteristics of Aquaponics through research and analysis of the data, and to think about how to improve it. The Aquaponics model is a farming method, in which plants are fed, fish are 
provided with a purified water quality environment, and they interact with each other through water circulation. It is mainly used for cultivation of large amount of fish and leafy vegetables.

It is environmentally friendly, because it does not use chemical fertilizers or pesticides. It produces crops and fish in the same space, which is profitable and has the advantage of reducing labor force. However, there is the disadvantage that it has high initial cost and the facility must be manually monitored. Although automatic control is introduced as a solution to this problem, it is at a rudimentary level, for example temperature control is performed by opening and closing the cultivation facilities. To overcome these problems, we considered a novel Aquaponics construction model and a manual IoT-based remote automation control system.

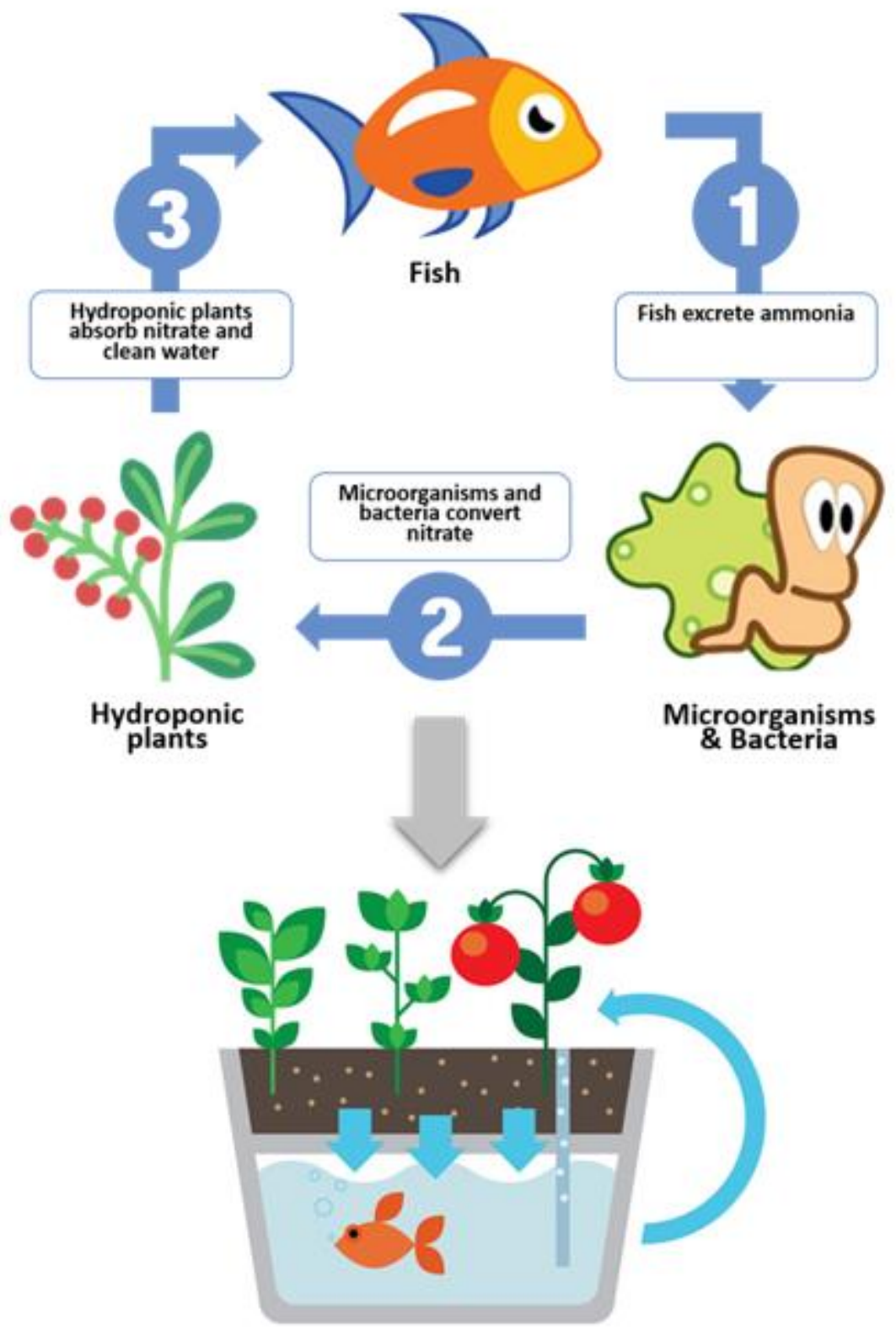

Figure 3. Aquaponics model 


\subsection{Modelling}

This step involves problem decomposition, abstraction, and algorithm design. We identified the key elements of the Aquaponics model at the problem decomposition and abstraction phase and expressed the system flow chart with a picture.

The Aquaponics model consisted in three steps, shown in Fig. 4. The first step involves establishing the optimal indoor cultivation environment. The second involves selecting the appropriate ornamental fish and flower garden. The third involves preparing for remote control by attaching sensors and actuators to a board with a microcontroller.

To build a growth and cultivation environment, we selected the fish tank with 15 liters of water and $32 \mathrm{~cm} \mathrm{x}$ $21 \mathrm{~cm} \times 33 \mathrm{~cm}$ in size, considering the cultivation area. We laid the Hydrobol so that the plant can chemically and biologically filter the nitrogen components dissolved in the water raised by the water pump. The amount of light, essential for plant cultivation, is very limited in the indoor environment. Hence, we used LED lamps to increase the amount of light. As plants we selected Watercoin, because it is easy to cultivate in hydroponics and the round leaves are suitable for decoration.

In this Aquaponics model, the control of growth and cultivation environment is an important factor to ensure that aquarium fishes and plants grow well. To this end, that the IoT is essential and it is necessary to collect data on the environment by temperature and light intensity sensors. To cope with environmental changes, we installed air pumps, fans, and water pumps as actuators on boards equipped with SoC (System on Chip) through 4-channel relay control. We planned to conduct control and monitoring through web applications from the Internet. Fig. 5 shows the design of the indoor Aquaponics model.
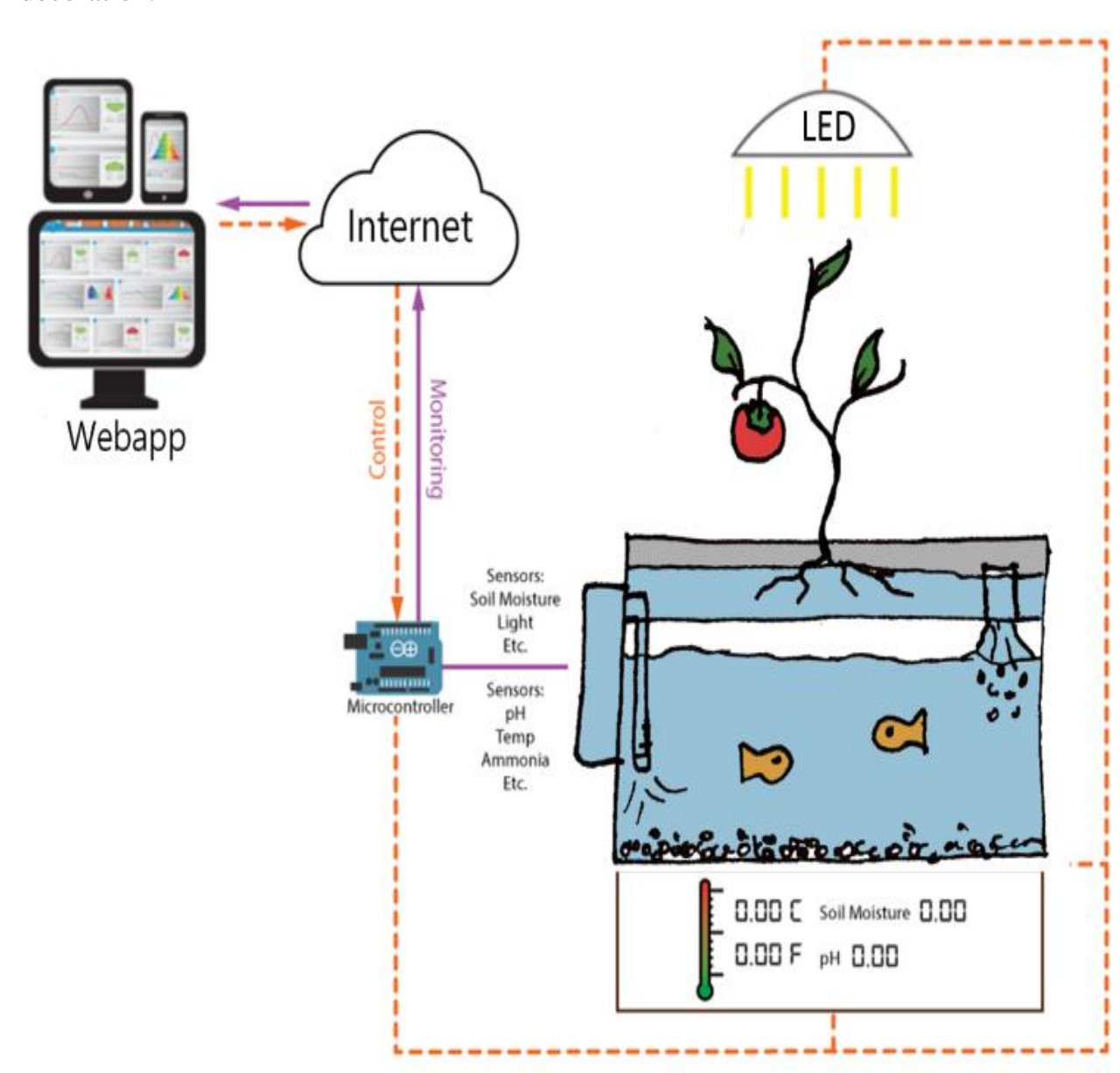

Figure 5. Design of indoor aquaponics model 
It is a phase of describing algorithms, which organize a series of steps performed to achieve a goal or to solve problems. The parameters that need to be controlled are the temperature and the amount of light. If the temperature rises above the one set for cultivation, the fan will operate. The amount of light is the most unstable parameter of the indoor environment due to artificial lighting. Therefore, the amount of light is measured by dividing it into the external environmental element and the internal environmental element, and LEDs will operate if the light amount falls below the set limit.

Fig. 6 is a flow chart of the system with boards, sensors, and actuators. The remote control system considered is developed from a web application as an interface. Fig. 7 shows the user interface of the system. It was programmed to monitoring the internal condition data (brightness, temperature, etc.) and the external condition data (brightness, etc.) through the sensors, and to control the actuator to provide the optimal growth environment.

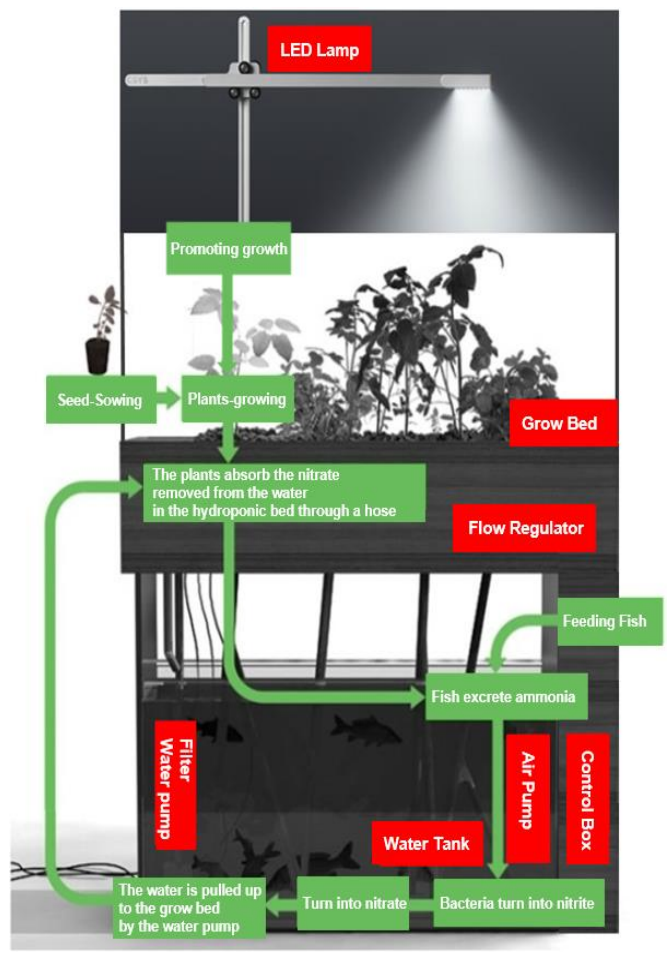

Figure 6. System overview
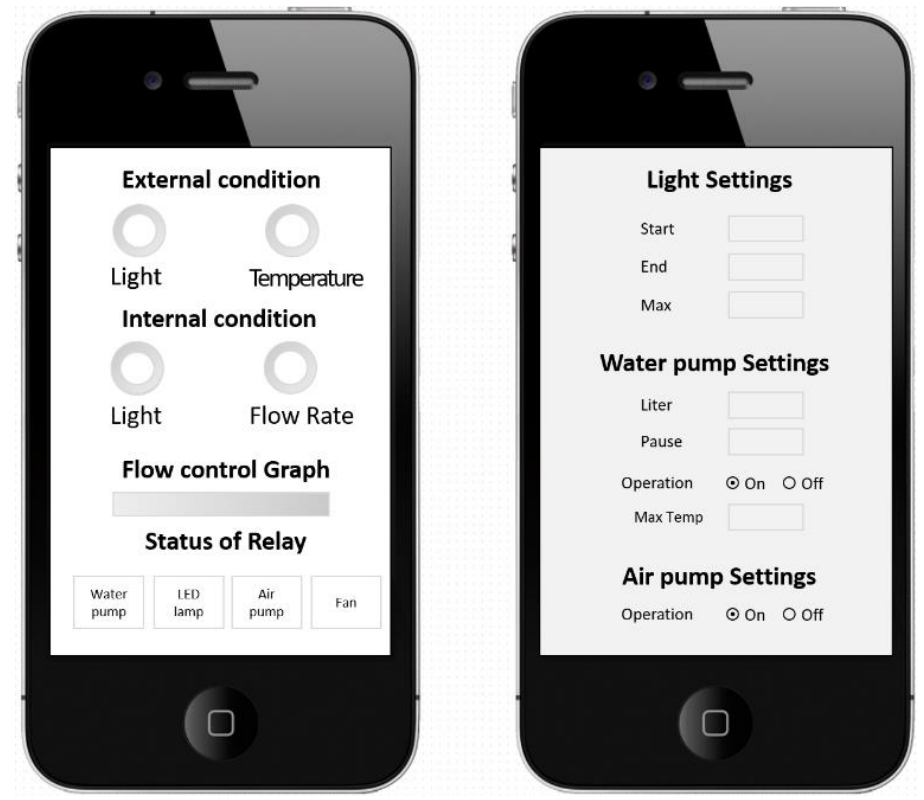

Figure 7. Remote control system user interface 


\subsection{Programming and Simulation}

In this step the Aquaponics model is implemented. The main components of the Aquaponics model are the following. We used Intel Edison Arduino Breakout Board as a microcontroller, and used a light intensity sensor, a LED for plant growth, a water pump, an air pump, and a power supply. The control system is based on Windows 10, uses HTML and CSS for UI creation, and uses JavaScript and jQuery for interaction. We also used Node.js for network application development and Intel XDK IoT for
IoT application development.

The microcontroller circuit configuration is shown Fig. 8. The power supplied through the AC input of $220 \mathrm{~V}$, controls the fan, air pump, LED, and water pump connected to each channel by the relay. In the sensor part, an external and internal light sensor, an external temperature sensor, and a flow rate sensor were used for sensing, and a resistance was applied to prevent excessive current in the sensors.

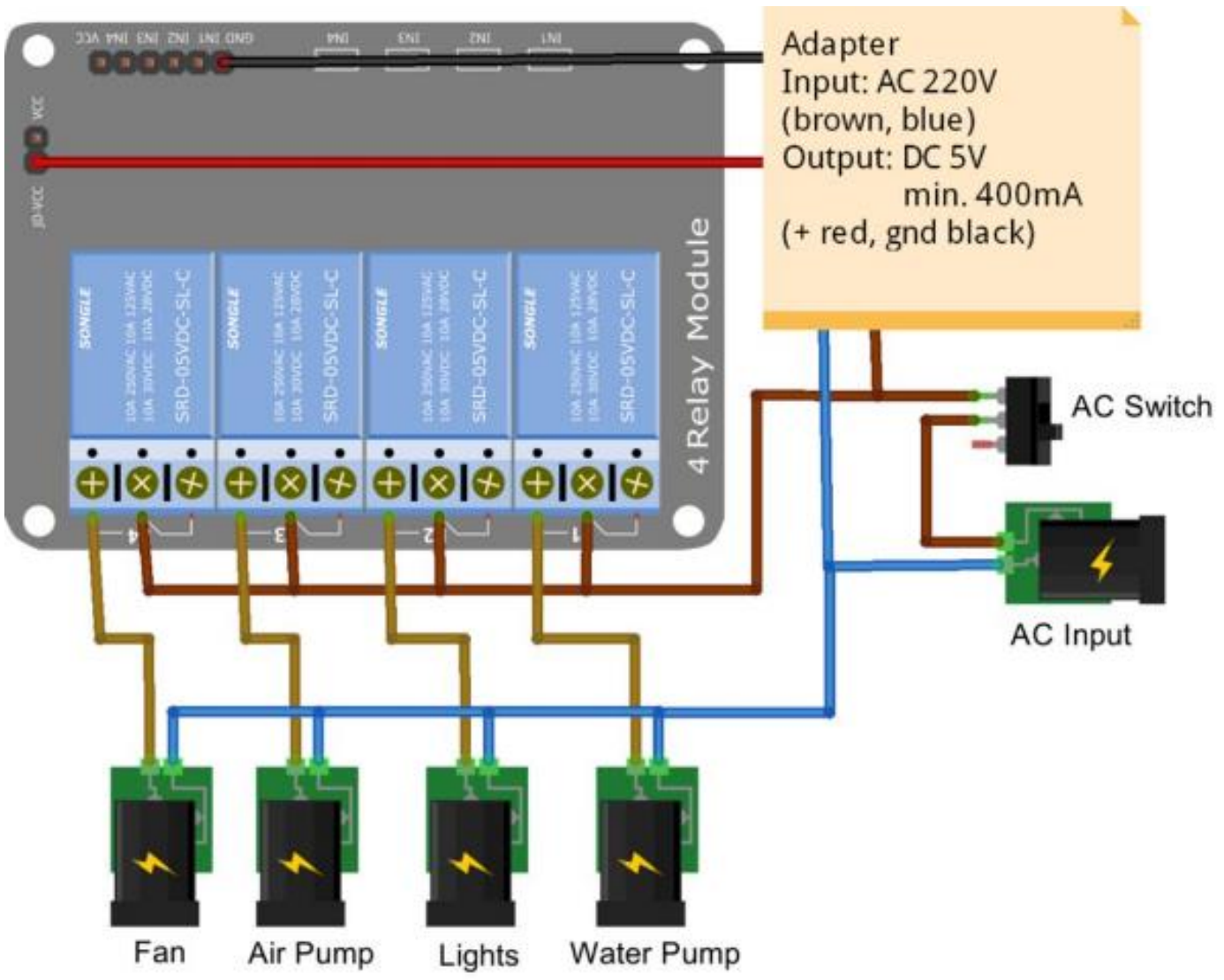




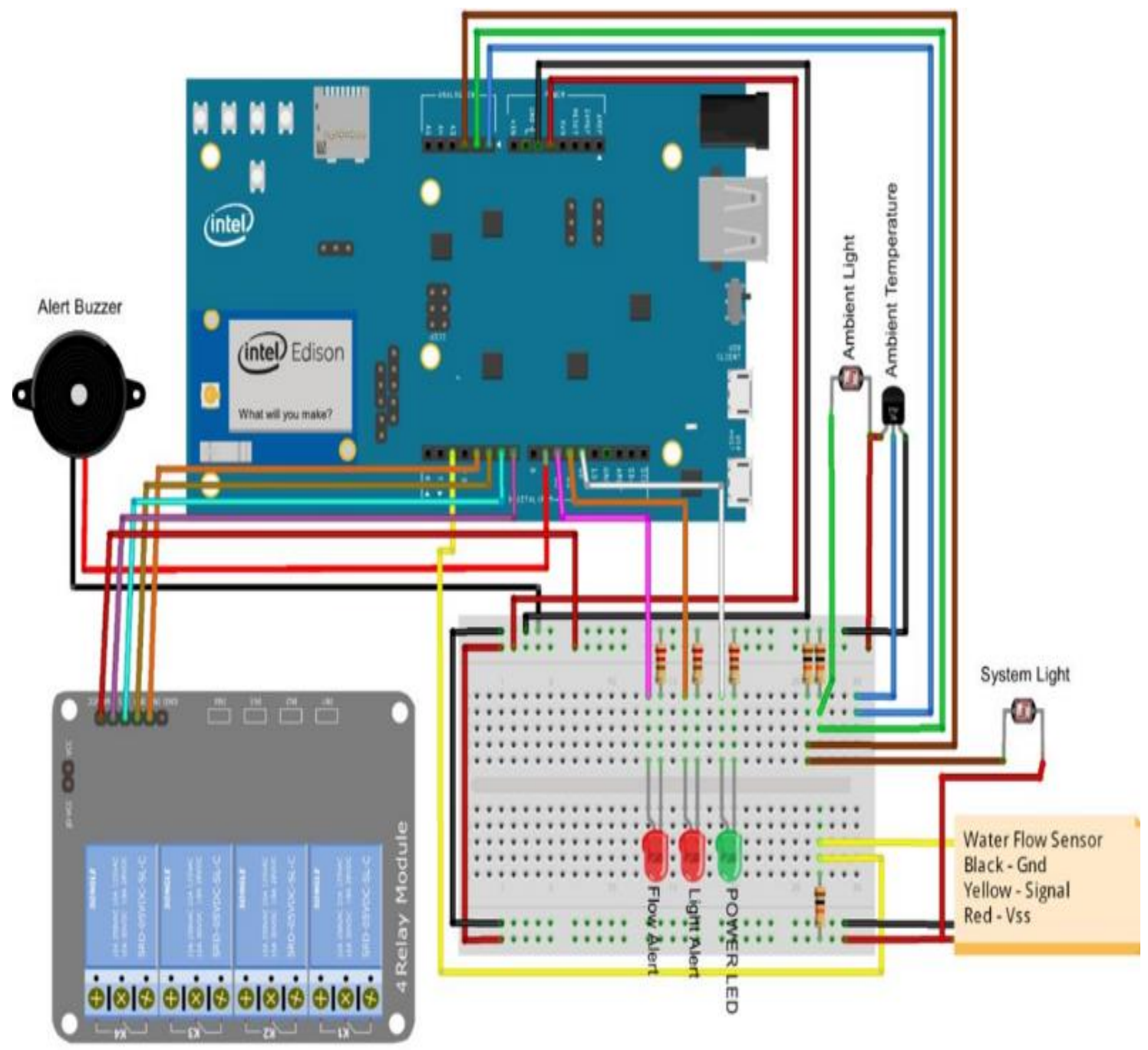

Figure 8. Microcontroller circuit

\subsection{Model Production}

In this step, the model is built. Fig. 9 combines an indoor Aquaponics model with a control system, both before and after the system is remotely operated.

When the power is supplied, the actuator is activated according to the set value. The developed remote-control system can be divided into the monitoring part and the control part. The operation result is shown Fig 10. In the monitoring part, light, temperature, and water flow, as well as the status of the 4-channel relay, can be monitored in real time. The power supply channel is changed to a green box, and the channel where no power is supplied is displayed as a red box. In the control part, there is one section to change the settings and one where data records can be viewed. The setting section is divided into four sub-sections: brightness setting, water pump setting, temperature and fan setting, and air pump setting. In the brightness setting section the start and end times, and the maximum brightness, can be set. 

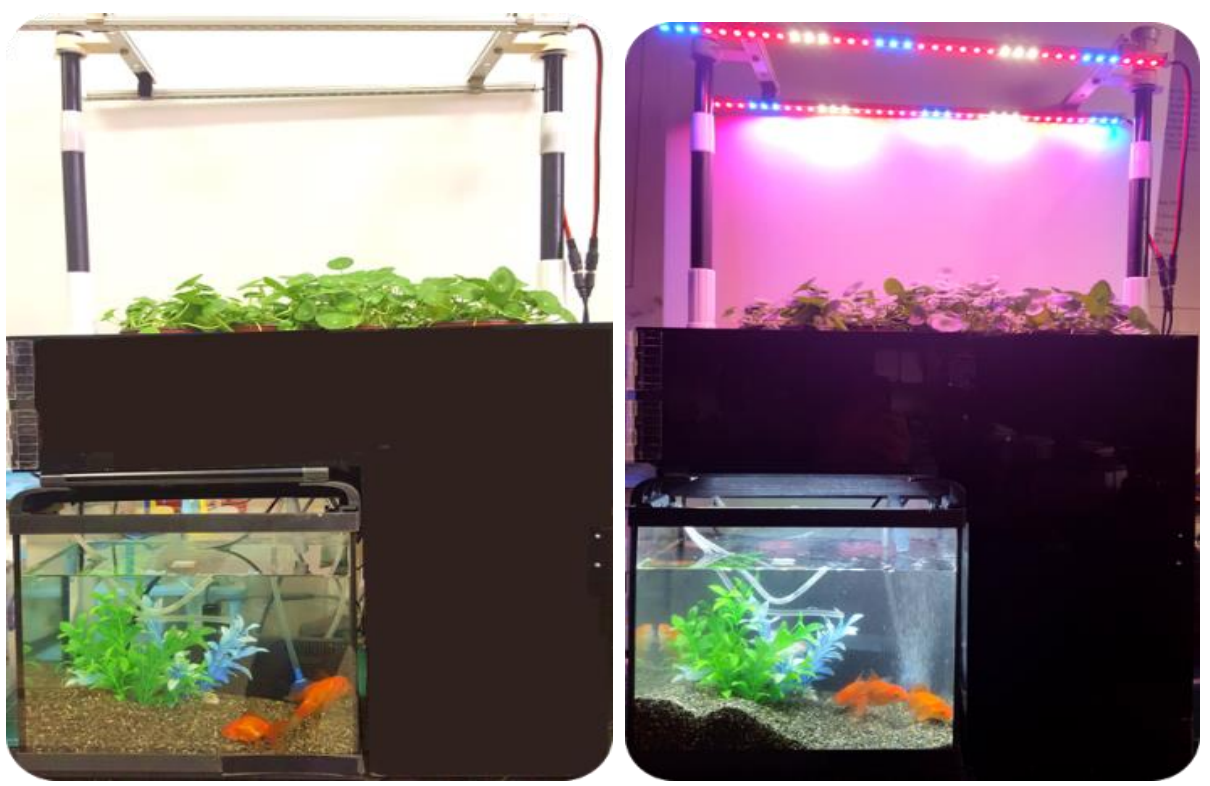

Figure 9. Model system before (up) and after (down) remote operation
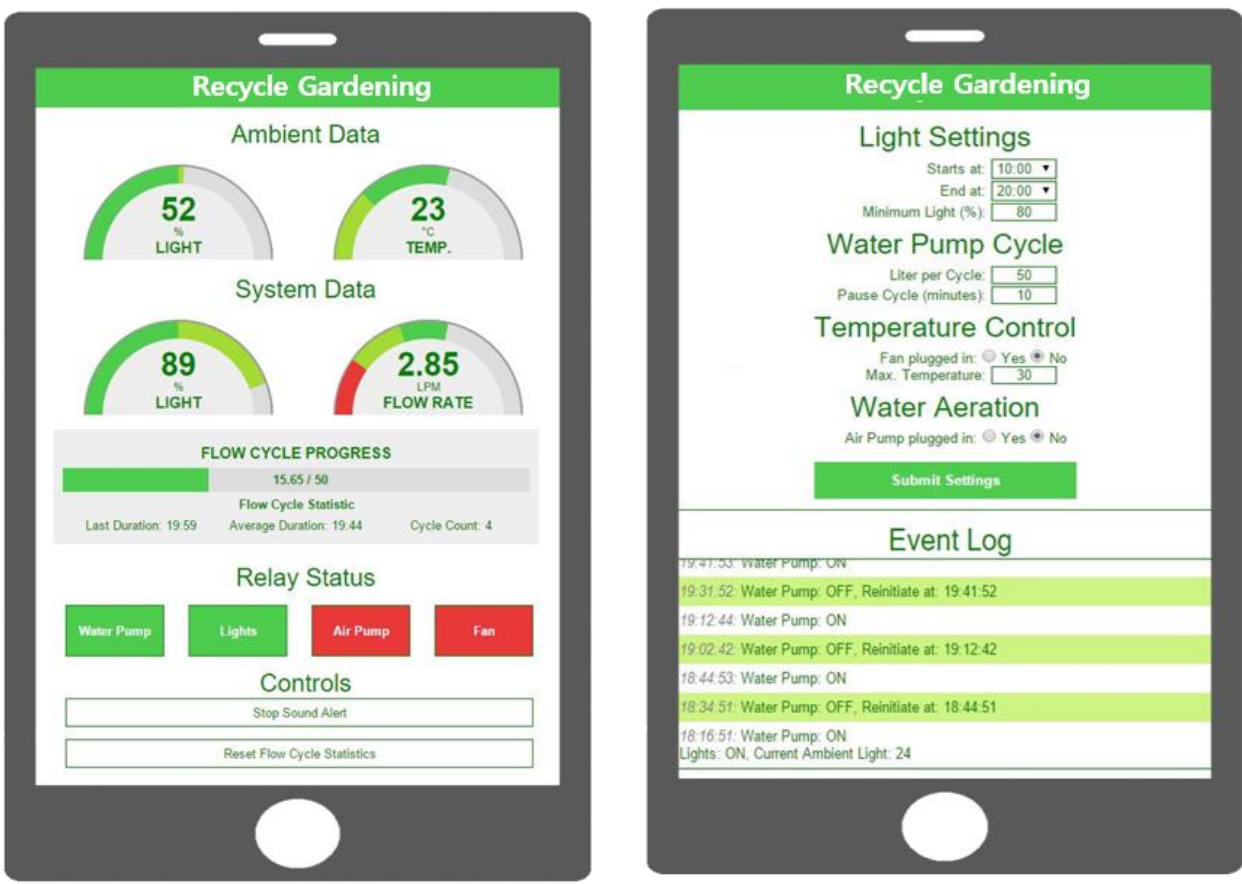

Figure 10. Program operation results

Table 2. Evaluation of Aquaponics system

\begin{tabular}{|c|c|}
\hline 1st implementation Test & 2nd implementation Test \\
\hline $\begin{array}{l}\text { - } \\
\text { - } \\
\text { - } \\
\text { Remote control using actuator } \\
\text { - } \\
\text { - } \quad \text { Recognition of supplementary items due to lack of light } \\
\text { Adequate water supply required for cultivation }\end{array}$ & 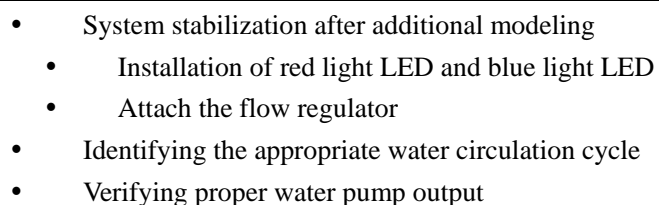 \\
\hline
\end{tabular}




\subsection{Evaluation and Generalization}

This is the last phase, where the Aquaponics system generated is evaluated and there is a discussions on what part of the system development was the most difficult and what knowledge was useful in the development process. Furthermore, improvements and applications of the system are discussed.

The first developed system enables remote control necessary for growth using environmental data generation, collection, monitoring, and actuator. However, there was a problem while observing whether the model stabilized. Watercoin requires a lot of sunlight, so white light LED lamps alone caused yellowing of the leaves over time due to lack of sunlight. It was difficult to find the cause of this problem, but with the help of experts in the field, we found that the amount of water circulating was constant, but the lack of light caused problems with the light saturation point. Thus, we installed a red and a blue light LED to solve this problem. TABLE 2 shows the evaluation process of the Aquaponics system.

As future developments of the system, the use of sensing technology such as oxygen saturation, electrical conductivity, and $\mathrm{pH}$ measurement to create an environment suitable for various plant cultivation were discussed. In addition, the Big Data analysis from the various plants was considered and we discussed the possibility of developing a situation aware control system.

The students who participated in the experiment claimed that they had a lot of difficulties, but as they were guided step by step by the model, they were able to gain pleasure and satisfaction from learning and seeing concepts, initially thought impossible, developed into a practical system.

\section{Conclusions}

In this study, we designed a CT-based convergence instructional model and developed an indoor Aquaponics control system using the designed model. The CT-based convergence instructional model was designed from the perspectives of $\mathrm{CT}$ and engineering thinking to find and solve various real-world problems. Through CT, the convergence of science, engineering and technology for creative problem solving can be achieved. Thus, the CT-based convergence instructional model is expected to enhance the ability to creatively solve real-world problems through fusion with STEM subject.

The CT-based convergence instructional model proposed in this study was applied to a small number of students during the gifted education program. To more accurately analyze the educational effects of this model, many experiments should be conducted for different students in the future.

\section{REFERENCES}

[1] K. Schwab, The Fourth industrial revolution: what it means, how to respond. World Economic Forum. Available: https://www.weforum.org/agenda/2016/01/the-fourth-indu strial-revolution-what-it-means-and-how-to-respond/

[2] H. Seo, "High Performance Implementation of SGCM on High-End IoT Devices", Journal of Information and Communication Convergence Engineering, vol. 15, pp. 212-216, 2017.

[3] Y. Hwang, K. Kim , O. Kwon, I. Moon, G. Shin , J. Ham , and J. Park, "Analyzing Box-Office Hit Factors Using Big Data: Focusing on Korean Films for the Last 5 Years", Journal of Information and Communication Convergence Engineering, vol. 15, pp. 217-226, 2017.

[4] Ministry of Education, Basic plans for activating software Education, Korea Press release, 2016.

[5] S. Choi, "Analysis if informatics' Curriculum from the Perspective of 21 st Century Skills and Computational Thinking," Journal of Korean Association of Computer Education, vol. 14, pp. 19-30, 2011.

[6] S. Psycharis, "STEAM in education: a literature review on the role of computational thinking, Engineering epistemology and Computational Science," Science Culture, vol. 2, pp. 51-72, 2018

[7] J. Sim, A. Lee, \& H. Kim, "Understanding and Solutions of STEAM Education and Convergence Talent Education in Korea," Journal of Korean Science Education Society, vol. 35, pp. 709-723, 2015.

[8] S. Ham, S. Lim, S. Park, \& K. Song, "Development of CT-STEAM Education Program Enhancing Integrated Thinking Skills for Elementary School," Journal of Korea Computer Education Society. Vol. 17, pp. 81-91, 2014.

[9] Y. Park, \& J. Hwang, "The foundation for the development and use of computing thinking practice tools Research," Journal of Korean Earth Science Society, vol. 10, pp. 140$160,2017$.

[10] J. Wing, "Computational thinking," Communications of the ACM, vol. 49, pp. 33-35, 2006.

[11] Kalelığlu, F., Gülbahar, Y., \& Kukul, V.,”A Framework for Computational Thinking Based on a Systematic Research Review. Baltic Journal of Modern Computing, vol. 4, pp.583-596.

[12] J. Wing, "Computational thinking and Thinking about Computing," Philosophical Transactions of the Royal Society, vol. 366, pp. 3717-3725, 2008.

[13] Dike, V. E., \& Dike, N. I. Youth Development and Conflict Resolution in Nigeria:" Assessment and Intervention Strategies". Asian Journal of Education and Training, 3(1), 30-42. 2017.

[14] Dogan, M. F., \& Dogan, Z. Identifying Common Errors in Vertical Lowercase Manuscript Writing of the First Graders in Primary School. Journal of Education and e-Learning Research, 5(3), 144-156. 2018.

[15] Dong, T., \& Qiu, L. Research on Self-Mentions and Author Identity in Academic English Writing. International Journal 
of English Language and Literature Studies, 7(4), 115-121. 2018.

[16] Ahmed U, Zin ML, Majid AH. Impact of Intention and Technology Awareness on Transport Industry's E-service: Evidence from an Emerging Economy. International Journal of Industrial Distribution \& Business (IJIDB). 2016, Vol. 30; 7(3):13-8.

[17] Dumbu, E. Promoting entrepreneurship through open and distance education in Zimbabwe. A case study of the Zimbabwe Open University students at Masvingo Regional Campus. International Journal of Business, Economics and Management, 1(6), 101-114. 2014.

[18] Eketu, C. A. Perspectives on Human Nature and Implications for Research in the Behavioural Sciences. International Journal of Emerging Trends in Social Sciences, 4(1), 42-46. 2018.

[19] Foulidi, X., Papakitsos, E. C., Papakitsos, E. C., Vamvakeros, X., \& Dimou, I. Teachers of Secondary Education and Professional Stress with Students Performance: A Geographical Case Study. Humanities and Social Sciences Letters, 6(3), 121-129. 2018.

[20] Parnornmast C, Jermsittiparsert K, Sriyakul T. Empirical discourse analysis on correlations between exchange rate and exports. Social and Management Research Journal. 2013, Vol; 10(2):39-51.

[21] Garba, A. S. Promoting Entrepreneurship Amidst Poverty and Unemployment: The Role of Nigerian Educational Policies. International Journal of Asian Social Science, 7(8), 684-695. 2017.

[22] K. Kim and K. Jang, "A Discussion on the Concert of Convergence for STEAM-Approach in Music Education," Journal of the Korea Elementary Education, vol. 26, pp. 211-234, 2015.

[23] Korea Science and Creativity Foundation. STEAM Education in Hands. Seoul: Korea Creative Foundation, 2012.

[24] National Research Council (U.S.). Report of a workshop of pedagogical aspects of computational thinking. Washington, D.C: National Academies Press, 2011.

[25] Jermsittiparsert K, Sriyakul T, Pamornmast C. Quality of Thai Media: Empirical Proposals on" Prime Minister's Eggs" Discourse. Asian Social Science. 2013, Vol. 1; 9(17): 218

[26] BBC. Introduction to computational thinking, Available https://www.bbc.com/bitesize/guides/zssk87h/revision/1

[27] K. Brennan \& M. Resnick, "Using artifact-based interviews to study the development of computational thinking in interactive media design," Paper presented at annual American Educational Research Association Meeting. 2012 Vancouver, Canada.

[28] C. Selby \& J. Woollard, "Refining an Understanding of Computational Thinking," 2014. Available: https://eprints.soton.ac.uk/372410 $\quad$ /1/3724 10 UnderstdCT.pdf

[29] Haseeb M, Abidin IS, Hye QM, Hartani NH. The impact of renewable energy on economic well-being of Malaysia: Fresh evidence from auto regressive distributed lag bound testing approach. International Journal of Energy Economics and Policy. 2018, Vol. 9 No. 1: 269-75.

[30] E. Lee, "STEAM Education Plan for Computational Thinking," Journal of Korea Computer Education Society, vol. 16, pp. 47-51, 2012.

[31] M. Park, The operational definition of computational thinking practices from the view of science education and its implication in science education, Thesis, Chosun University, 2017

[32] S. Han \& M. Ryu, Software education for Computational thinking, Seoul: Saeng Ryeng Publishment, 2017. 